\title{
Modeling of Natural Lighting Parameters in the Open Air with Intermeradiandiate Luminance Distribution
}

\author{
Evgeniy Konopatskiy ${ }^{1}$, Vladimir Yehorchenkov ${ }^{2}$ and Andrey Bezditnyi ${ }^{3}$ \\ ${ }^{1}$ Donbas National Academy of Civil Engineering and Architecture, Derzhavina Street, 2, Makeevka, 286123, \\ Ukraine \\ ${ }^{2}$ Kyiv National University of Construction and Architecture, Povitroflotsky Avenue, 31, Kyiv, 03037, Ukraine \\ 3 Sevastopol branch of «Plekhanov Russian University of Economics», Vakulenchuk Street, 29, Sevastopol, \\ 299053, Russian Federation
}

\begin{abstract}
When simulating outdoor natural lighting, a spherical sky model is used. This is true in a clear sky, but in the presence of clouds, this model does not correspond to reality. This paper presents the substantiation of the sky model in the form of a spherical segment with a standard distribution of the luminance of the semi-clear (intermediate) sky. Moreover, instead of the ratio of illumination, under given cloudiness conditions to illumination with ideal transparency of the atmosphere, which are usually used in European standards, the direct value of cloudiness is used here, taken from the results of long-term observations at meteorological stations. To modeling the parameters of outdoor natural lighting, a more effective and simple mathematical apparatus of point calculus is used, with the help of which a point set of a spherical segment is formed. On the basis of this set, a field of elementary pyramids is created. For each pyramid, using well-known formulas, elementary values of the parameters of natural lighting are determined.
\end{abstract}

\section{Keywords}

Outdoor natural lighting, semi-clear sky, cloudiness, luminance distribution, sky model, spherical segment, point calculus, geometric model.

\section{Introduction}

In most studies and recommendations on the luminance distribution over the sky and on the determination of external illumination, a hemisphere was taken as a model of the sky [1-5]. This is true for a clear (Fig. 1) sky, but as soon as cloudiness is entered into the sky model, this model is not suitable for a semi-clear (intermediate) sky.

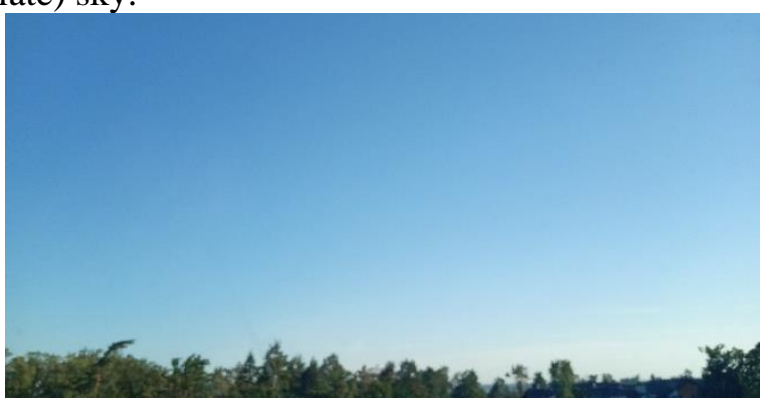

Figure 1: Clear sky

Most cloud types have a flat base at a certain height, which is characteristic of this type of cloud. At this height, the air that has risen from the ground level cools down to the dew point temperature, at which it can no longer cope with the water vapor contained in it. At this temperature, water vapor

GraphiCon 2021: 31st International Conference on Computer Graphics and Vision, September 27-30, 2021, Nizhny Novgorod, Russia EMAIL: e.v.konopatskiy@mail.ru (E. Konopatskiy); egval@ukr.net (V. Yehorchenkov); bezdytniy@gmail.com (A. Bezditnyi) ORCID: 0000-0003-4798-7458 (E. Konopatskiy); 0000-0003-2910-0331 (V. Yehorchenkov); 0000-0003-0528-9731 (A. Bezditnyi) (c) (i) 2021 Copyright for this paper by its authors.

Use permitted under Creative Commons License Attribution 4.0 International (CC BY 4.0).

CEUR Workshop Proceedings (CEUR-WS.org) 
condenses and forms droplets of liquid water, which we see as a cloud. For different types of clouds, the height of their location is different [6]. For example, low-level clouds are located at an altitude of up to $2000 \mathrm{~m}$ (Fig. 2). Middle-level clouds (Fig. 3), which include cumulus, stratocumulus, cumulonimbus, are located at an altitude of 2000 to $5000 \mathrm{~m}$. High-level clouds - nimbostratus, altostratus and altocumulus clouds - are located at an altitude of $5000 \mathrm{~m}$, etc. For example, in Figures 3 and 4 show stratocumulus and cumulus clouds (all photos are copyright).

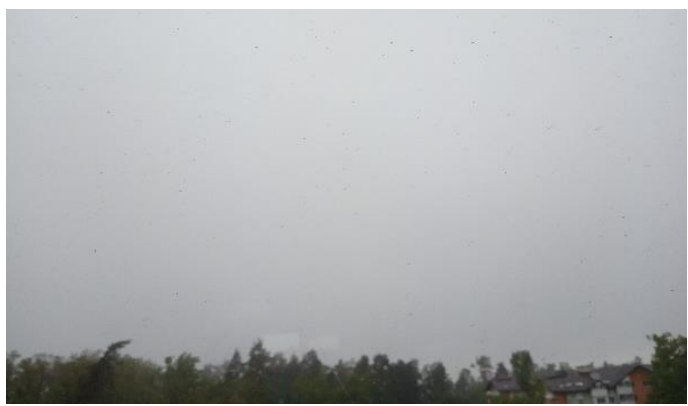

Figure 2: Overcast sky

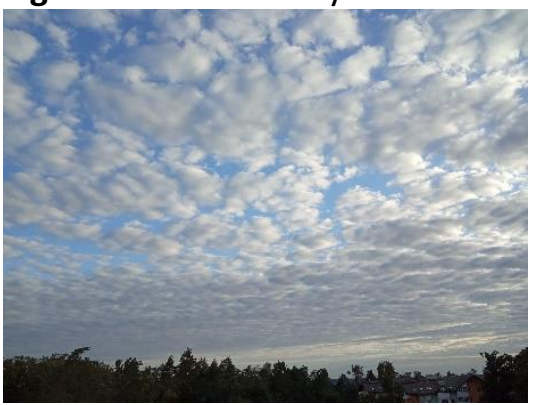

Figure 4: Location of cumulus clouds

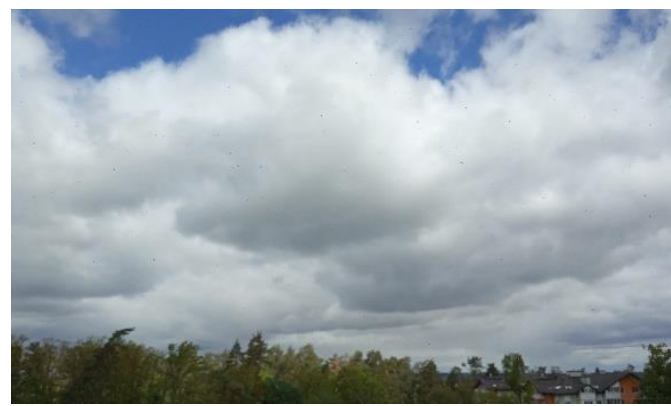

Figure 3: Stratocumulus clouds

The spherical shape of the firmament does not reflect reality in the transition to the assessment of the parameters of natural lighting on the semi-clear sky model (Fig. 5a). This can be seen from the figures 3 and 4 real examples.

The bases of the clouds are located parallel to the earth's surface, so the calculation scheme when determining the light and climatic parameters will look as shown in Fig. 5b.

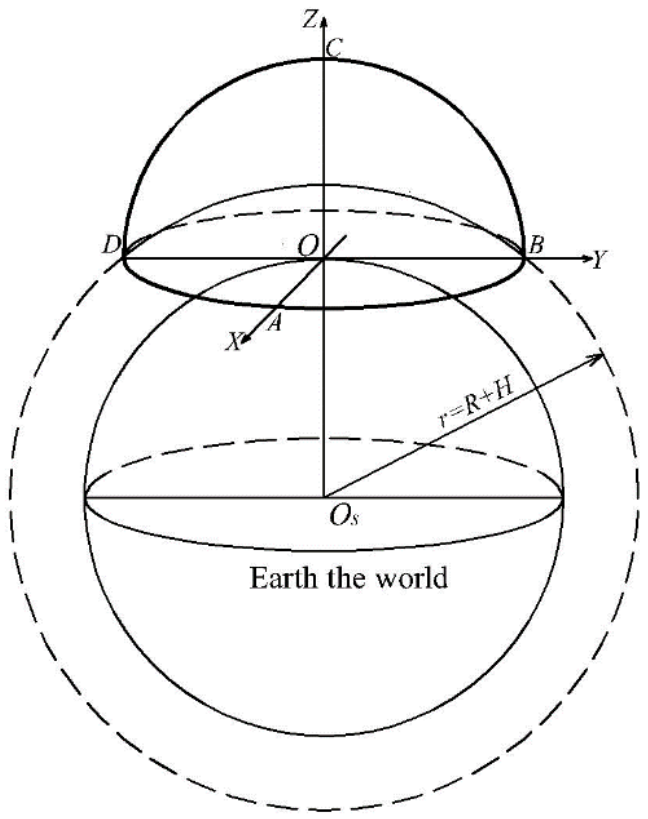

a)

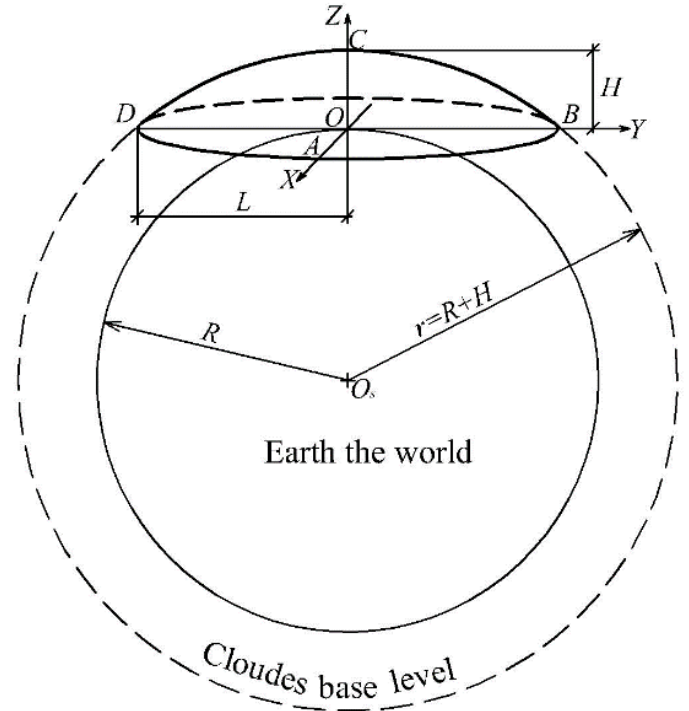

b)

Figure 5: Geometric comparison of the sky models: a - spherical; b - in the form of a spherical segment 
Many researchers have been engaged in models of the distribution of brightness and illumination from different types of the firmament [1-5]. A large number of experimental studies have been carried out on the distribution of brightness over the sky, including intermediate (semi-clear) $[4,5,7,8]$. Based on the results of these studies, a classification of sky types was carried out [9] according to combinations of regression coefficients that characterize the indicatrix of light scattering in the atmosphere. There are two points to note here. Regression coefficients and their combinations are special cases of physical models. The parameter characterizing cloudiness is not the direct value of cloudiness, but the ratio of scattered radiation to total radiation.

The purpose of this work is to modeling outdoor natural lighting with a semi-clear (intermediate) distribution of the sky luminance, as well as to develop a method for calculating the parameters of the light field using the mathematical apparatus of point calculus. This will make it possible to form a more realistic picture of natural lighting in rooms with light openings of any shape, which are arbitrarily located in space.

To achieve this goal, you must complete the following tasks:

1. Construct a geometric model of the luminance distribution of the semi-clear sky and determine its computational algorithm based on point equations.

2. To improve the methodology for determining the main parameters of outdoor lighting, taking into account the refined geometric model of the semi-clear sky luminance distribution.

\section{Geometric model of the semi-clear sky luminance distribution}

To solve the set tasks, we present a diagram of a spherical segment with interconnected parameters (Fig. 7) [10]:

$$
L=\sqrt{H(2 r+H)},
$$

where $r$ is the radius of the full sphere from the center of the Earth to the base of the location of the clouds, $\mathrm{km}$;

$L$ is the distance of the horizon line from the center $O$ (observer), km;

$H$ is the height of the spherical segment from the center $O$ to its zenith $C, \mathrm{~km}$.

The ratio of the base height for different types of clouds to the distance from the observer to the horizon line (Fig. 6) is shown in the table 1.

Table 1

The ratio of the height of the clouds and the horizon

\begin{tabular}{cccccccccc}
\hline $\begin{array}{c}\text { Location } \\
\text { height, } H, \\
\text { km }\end{array}$ & 2 & 3 & 4 & 5 & 6 & 7 & 8 & 9 & 10 \\
\hline $\begin{array}{c}\text { Horizon, } L, \\
\text { km }\end{array}$ & 159,64 & 195,52 & 225,78 & 252,44 & 276,54 & 298,71 & 319,35 & 338,73 & 357,07 \\
\hline
\end{tabular}

As can be seen from Figure 6, the cloud base is located almost horizontally (parallel to the earth's surface) over a large extent. Many geometric and physical characteristics change with a small curvature of the sky.

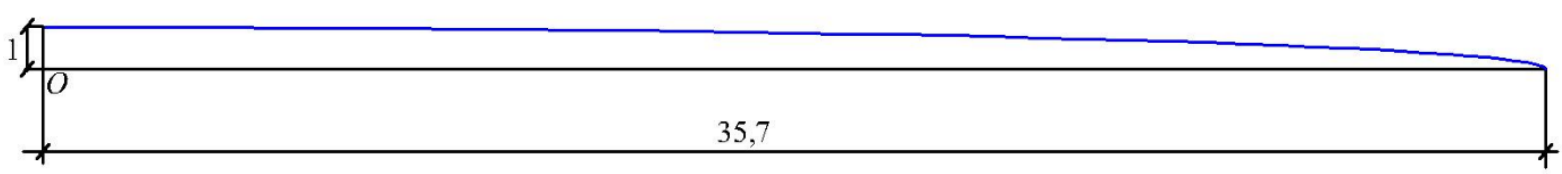

Figure 6: The real shape of the semi-clear (intermediate) sky at maximum curvature $(H=10 \mathrm{~km})$

For the convenience of calculations, we use the mathematical apparatus of point calculus [11-15].

A feature of the point calculus is the analytical description of geometric objects in the form of simple point equations and computational algorithms that are invariant with respect to parallel projection. This 
allows, within the framework of affine geometry, to obtain a generalization to the multidimensional space.

The generalized equation of an ellipsoid in point form was obtained by O.A. Chernyshova [16]. Here we use this equation for a point description of the particular case of a spherical segment.

$$
M_{i j}=(A-O) \cos \varphi_{i} \cos \beta_{j}+(B-O) \cos \varphi_{i} \sin \beta_{j}+(C-O) \sin \varphi_{i}+O .
$$

Or in coordinate form:

$$
\left\{\begin{array}{l}
x_{i j}=\left(x_{A}-x_{O}\right) \cos \varphi_{i} \cos \beta_{j}+\left(x_{B}-x_{O}\right) \cos \varphi_{i} \sin \beta_{j}+\left(x_{C}-x_{O}\right) \sin \varphi_{i}+x_{O} ; \\
y_{i j}=\left(y_{A}-y_{O}\right) \cos \varphi_{i} \cos \beta_{j}+\left(y_{B}-y_{O}\right) \cos \varphi_{i} \sin \beta_{j}+\left(y_{C}-y_{O}\right) \sin \varphi_{i}+y_{O} ; \\
z_{i j}=\left(z_{A}-z_{O}\right) \cos \varphi_{i} \cos \beta_{j}+\left(z_{B}-z_{O}\right) \cos \varphi_{i} \sin \beta_{j}+\left(z_{C}-z_{O}\right) \sin \varphi_{i}+z_{O} .
\end{array}\right.
$$

where $\varphi$ is the angular height of the current point of a circle of an arbitrary section (Fig. 7);

$\beta_{j}$ is the horizontal angle of the current point from some fixed position (for example, north).

This point equation of a segment in the $A B C D$ simplex with two current parameters $\varphi$ and $\beta$ (azimuth) uniquely determines the position of the current point $M_{i j}$ on the segment surface (Fig. 7)

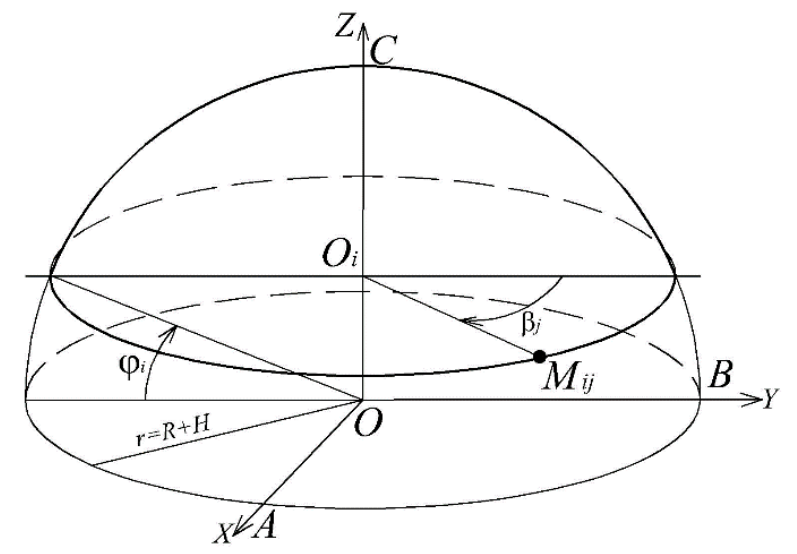

Figure 7: Parameters of a spherical segment in a simplex $A O B C$

The visualization of the obtained point equation (3) is carried out in the Maple environment in the following ranges: $\beta=0 \ldots .2 \pi, \varphi=\arcsin \left(1-\frac{H}{r}\right) \ldots \frac{\pi}{2}$.

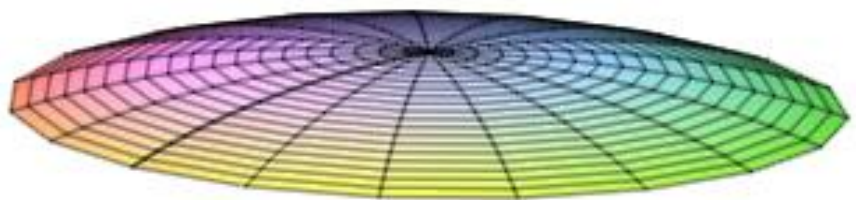

Figure 8: Visualization of spherical segment scanning points in one of the computer algebra systems (scanning points are located at the intersection of parallels and meridians)

\section{Method for determining the main parameters of outdoor lighting}

The luminous flux in the center of the spherical segment from its entire surface is formed within the solid angle with the apex at this point. If we use the coordinates of the obtained scanning points from equation (3), then the entire solid angle of a spherical segment can be represented as the sum of elementary solid angles (pyramids) with apex at point $O_{i}$, and the pyramid faces pass through four adjacent scanning points (Fig. 9). The base of the pyramids is an elementary surface area of a spherical segment. 


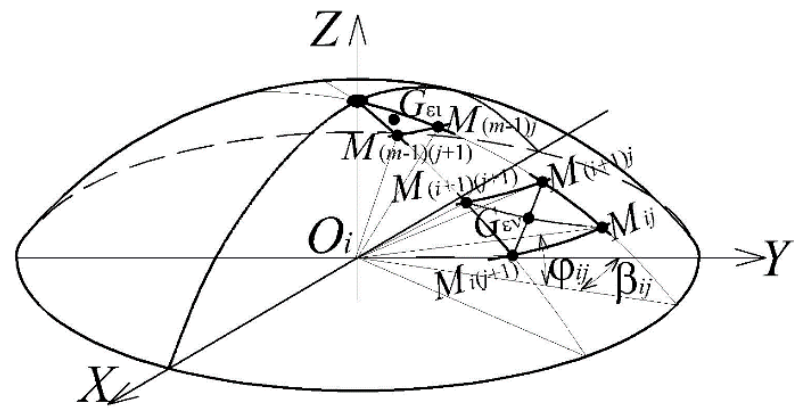

Figure 9: Formation of elementary solid angles in a spherical segment of the sky

We can assume that within an elementary solid angle (elementary pyramid), the luminance of the sky is constant, and its value corresponds to the luminance value in the direction from the center of the spherical segment to the center of elementary pyramid base gravity (we will assume that for an elementary quadrangle - the middle of one of the diagonals).

Then the coordinates of the center of gravity for the quadrangular element:

$$
\begin{gathered}
G_{\varepsilon v}=\frac{M_{i j}+M_{(i+1)(j+1)}}{2} ; \\
x_{\varepsilon v}^{G}=\frac{x_{i j}+x_{(i+1)(j+1)}}{2} ; \quad y_{\varepsilon v}^{G}=\frac{y_{i j}+y_{(i+1)(j+1)}}{2} ; \quad z_{\varepsilon v}^{G}=\frac{\mathrm{z}_{i j}+\mathrm{z}_{(i+1)(j+1)}}{2} ;
\end{gathered}
$$

for triangular element:

$$
\begin{gathered}
G_{\varepsilon 1}=\frac{M_{(m-1) j}+M_{(m-1)(j+1)}+C}{3} ; \\
\left\{\begin{array}{l}
x_{\varepsilon \mathrm{l}}^{G}=\frac{x_{(m-1) j}+x_{(m-1)(j+1)}+x_{C}}{3} \\
y_{\varepsilon \mathrm{l}}^{G}=\frac{y_{(m-1) j}+y_{(m-1)(j+1)}+y_{C}}{3} \\
z_{\varepsilon \mathrm{l}}^{G}=\frac{\mathrm{z}_{(m-1) j}+\mathrm{z}_{(m-1)(j+1)}+\mathrm{z}_{C}}{3}
\end{array}\right.
\end{gathered}
$$

The horizontal illumination in the center of the spherical segment, $\Delta E_{\mathrm{k}}$, from a section of the sky within the framework of an elementary quadrangular pyramid is determined by the formula:

$$
\Delta E_{\varepsilon v}=L_{z} g_{\varepsilon v}^{G} \Delta \sigma_{\varepsilon v}^{G} \cos \gamma
$$

where $L_{z}$ is the luminance of the sky at the zenith, $\mathrm{cd} / \mathrm{m}^{2}$;

$g_{\varepsilon v}^{G}$ is the distribution of relative luminance over the sky $[9,17]$.

To determine this parameter, one can use the proposal of Gillete G. Pe., Treado S. [18], according to which the luminance of the semi-clear (intermediate) sky $L_{S / I}$ is determined by the following formula:

$$
L_{S / I}=L_{C} t+L_{C S}(1-t) \text {, }
$$

where $L_{\mathrm{C}}$ and $L_{\mathrm{CS}}$ are the luminance at a given point in the sky, respectively, for a clear and cloudy sky, $\mathrm{cd} / \mathrm{m}^{2}$

$t$ is the cloud dependent parameter. 


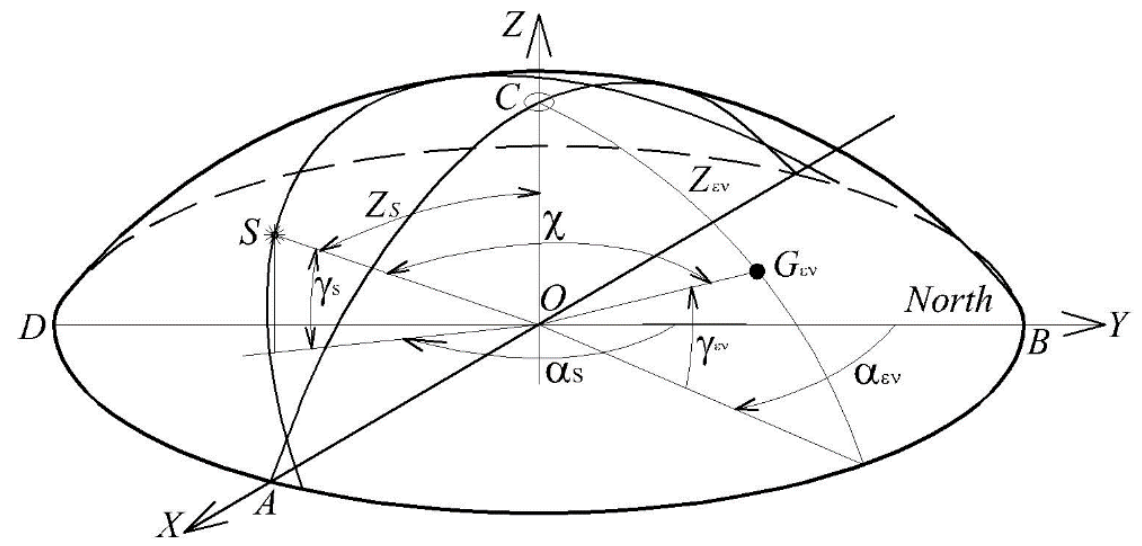

Figure 10: Angular coordinates of the Sun and this element of the sky

The distribution of the relative luminance over the sky, taking into account [17], will have the form:

$$
g_{\varepsilon v}^{G}=\frac{L_{\varepsilon v}}{L_{z}}=\frac{t\left(1+c_{1}\left[e^{d_{1} \chi}-e^{d_{1} \frac{\pi}{2}}\right]+e_{1} \cos ^{2} \chi\right)\left(1+a_{1} e^{\frac{b_{1}}{\cos Z}}\right)}{\left(1+c_{1}\left[e^{d_{1} Z_{S}}-e^{d_{1} \frac{\pi}{2}}\right]+e_{1} \cos ^{2} Z_{S}\right)\left(1+a_{1} e^{b}\right)}+\frac{(1-t)\left(1+a_{2} e^{\frac{b_{2}}{\cos Z}}\right)}{1+a_{2} e^{b}},
$$

where $t=\frac{1+\cos \left(K_{\mathrm{o}} \cdot \pi\right)}{2}$;

$K_{\mathrm{o}}$ is the cloudiness coefficient in fractions of a unit, adopted on the basis of long-term observations of cloudiness at meteorological stations [19]. At its minimum values, the sky approaches a clear state ( $K_{\mathrm{o}}=0$ only at the outer boundary of the atmosphere. When $K_{0}=1$ cloudy sky is observed (overcast standard cloudy sky). This coefficient is proposed to be used instead of the ratio of illumination under given cloudiness conditions to illumination with ideal atmospheric transparency, which is usually used in European standards.

The firmament is taken as the first term, for example, of the type 13: $a_{1}=-1, b_{1}=0.32, c_{1}=16$, $d_{1}=-3, e_{1}=0.3-$ standard cloudless sky according to CIE, polluted atmosphere [9];

The firmament is taken as the second term, for example, of the type 3: $a_{2}=1,1, b_{2}=-0,8, c_{2}=0$, $d_{2}=-1, e_{2}=0-$ standard cloudy sky according to CIE, polluted atmosphere;

$\Delta \sigma_{\varepsilon v}^{G}$ - the value of the projection of the vector of the solid angle of the elementary pyramid on the normal to the calculated plane, which is determined by the above method, is determined using a wellknown formula [20]:

$$
\Delta \sigma_{\varepsilon v}^{G}=\frac{1}{2} \sum_{\lambda=1}^{p} \alpha_{i j} \cdot \cos \beta_{i j},
$$

the parameters included in this formula are shown in Fig. 11;

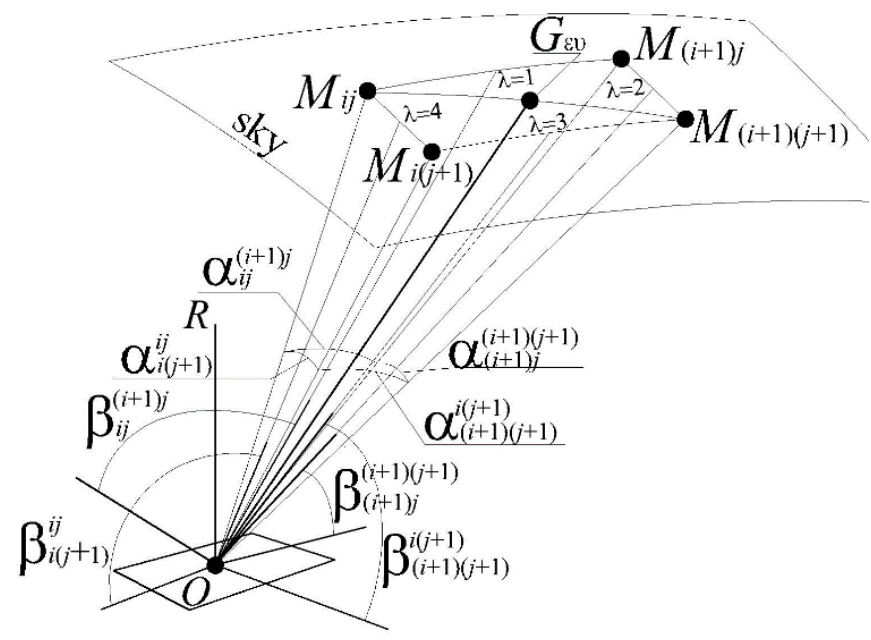

Figure 11: Geometric parameters of the elementary solid angle 
$\gamma$ - the angle between the normal to the base of the elementary pyramid and the axis of the solid angle (Fig. 12).

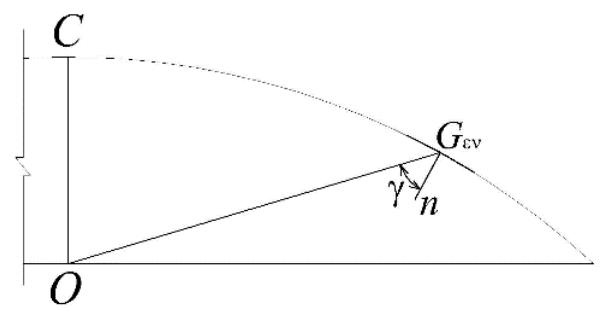

Figure 12: The angle between the normal to the base of the pyramid and the axis of the solid angle

An effective parameter that can be used to study the light climate and develop light-climatic maps is the average spherical illumination, which most fully reflects the light resources of a given area. It takes into account the reflected component from the earth's surface. Direct accounting of this component when using horizontal illumination is impossible.

Elementary average spherical illumination in the center of a spherical segment $\Delta E_{\varepsilon v}^{4 \pi}$, within an elementary solid angle is determined by the following formula [20]:

where $\Delta \omega_{\varepsilon v}$ is the elementary solid angle.

$$
\Delta E_{\varepsilon v}^{4 \pi}=0,25 g_{\varepsilon v} L_{z} \Delta \omega_{\varepsilon v}
$$

The solid angle is determined by the following formula [21]:

$$
\Delta \omega_{\varepsilon v}=\left(\sum_{\lambda=1}^{p} A_{\lambda, \lambda+1}\right)-2 \pi,
$$

where $p$ is the number of faces of an elementary pyramid;

$A_{\lambda, \lambda+1}$ is the value of the internal dihedral angle between the planes that pass through the center of the spherical segment and the $\lambda$-th and $\lambda+1$-th faces of the elementary pyramid (Fig. 13). In determining this angle, a technique developed in point calculation is used [17].

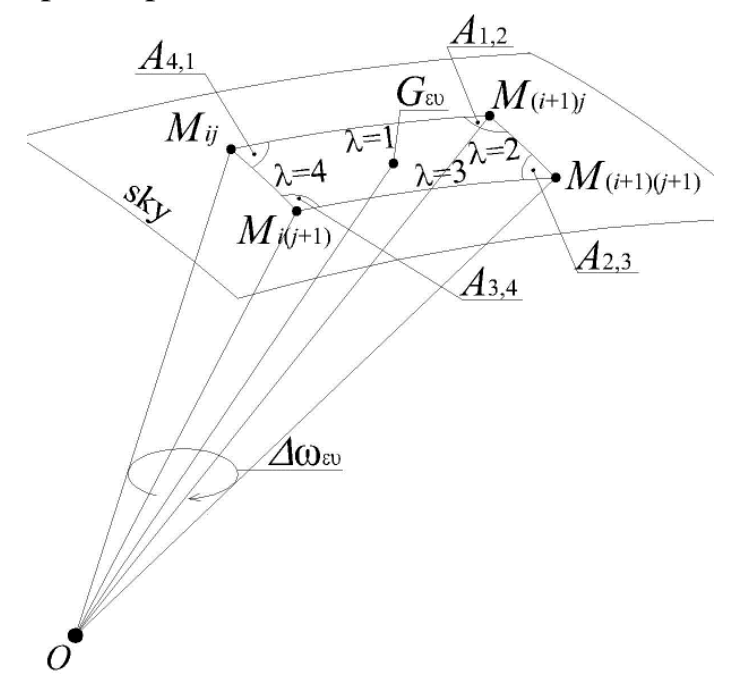

Figure 13: Scheme for determining the value of the elementary solid angle

\section{Conclusion}

In this paper, we propose and substantiate the use of a geometric model of the sky in the form of a spherical segment with a standard distribution of the semi-clear sky luminance (intermediate). At the same time, the following results were obtained, which have scientific novelty and practical value:

1. A geometric model of a semi-clear sky has been constructed, which is distinguished by the fact that instead of a spherical model, it is proposed to use a model in the form of a spherical segment. The segment is at the level of the cloud base and is bounded from below by a plane passing through the observation point and the visible horizon line, which better corresponds to reality. 
2. The distribution of luminance over the sky has been clarified, at which it is proposed to use cloudiness in fractions of a unit as the cloudiness coefficient. It was adopted on the basis of long-term observations at meteorological stations instead of the ratio of illumination under given cloudiness conditions to illumination with ideal atmospheric transparency, which is usually used in European standards.

3. The method for determining the main parameters of outdoor lighting has been improved: horizontal and average spherical illumination, taking into account the refined geometric model of the semi-clear sky luminance distribution.

\section{References}

[1] J. Mardaljevic, Daylight Simulation: Validation, Sky Models and Daylight Coefficients, De Montfort University, Leicester, 1999.

[2] P. Tregenza, Analyzing sky luminance scans to obtain frequency distributions of CIE Standard General Skies, Lighting Res. Technol, 1987, No. 19 (1), pp. 13-14.

[3] S. Darula, Review of the current state and prospects of standardization in the field of natural indoor lighting, Lighting engineering, 2019, No. 1, pp. 6-20.

[4] N. Igawa, H. Nakamura, K. Matsuura, Sky luminance distribution model for simulation of daylit environment, Proceedings of IBPSA Conference, 1999, Vol. 2, pp. 969-975.

[5] R. Perez, R. Seals, J. Michalsky, All weather model for sky luminance Distribution-preliminary configuration and validation, Solar Energy, 1993, Vol. 50, No. 3, pp. 235-245.

[6] A New Edition of the International Cloud Atlas, WMO BULLETIN, Vol. 66 (1), 2017.

[7] K. Kaneda, T. Okamoto, E. Nakame, T. Nishita, Photorealistic image synthesis for outdoor scenery under various atmospheric conditions, The Visual Computer 7, 5 and 6, 1991, pp. 247-258.

[8] H. Nakamura, M. Oki, Y. Hayashi, Luminance distribution of Intermediate Sky, J. Light \& Vis. Env, Vol. 9, No. 1, 1985, pp. 6-13.

[9] ISO 15469: 2004, Spatial distribution of daylight - CIE standard general sky.

[10] S.V. Zvereva, In the world of sunlight, Gidrometeoizdat, Leningrad, 1988.

[11] I. G. Balyuba, E. V. Konopatskiy, A. I. Bumaga, Point calculus, Makeevka, 2020.

[12] V.A. Egorchenkov, E.V. Konopatsky, Principles of constructing light field model for a room with curvilinear quadrangular light openings by means of the dot calculation, Light \& Engineering, Vol. 23, No. 2, pp. 43-48, 2015.

[13] E.V. Konopatskiy, A.A. Bezditnyi, Ya.A. Kokareva, V.V. Kucherenko, Features visualization of geometric objects in the BN-calculus, Scientific Visualization, Vol. 12, No. 2, pp. 98-109, 2020. doi: $10.26583 / \mathrm{sv} .12 .2 .08$.

[14] E.V. Konopatskiy, A.A. Bezditnyi, A.I. Litvinov, Geometric modeling of torse surfaces in BNcalculus, IoP conference series: Journal of Physics: Conf. Series 1791 (2021) 012050. doi: 10.1088/1742-6596/1791/1/012050.

[15] E.V. Konopatskiy, A.A. Bezditnyi, M.V. Lagunova, A.V. Naidysh, Principles of solid modelling in point calculus, IoP conference series: Journal of Physics: Conf. Series 1901 (2021) 012063. doi: 10.1088/1742-6596/1901/1/012063.

[16] O.A. Chernysheva, Krasovsky's ellipsoid in point calculus of Baluba-Naidysh, Bulletin of the Donbass National Academy of Civil Engineering and Architecture, Makeevka, Issue 2014-4(108), 2014, pp. 6-9.

[17] V.A. Yehorchenkov, Scientific bases of formation of the comfortable environment in buildings on dynamic parameters of natural lighting, Kyiv, 2019.

[18] G.Pe. Gillete, S. Treado, The issue of sky conditions, Lighting Design and Application, March 1985.

[19] Scientific and applied reference book on the climate of the USSR, Series 3, long-term data, Parts 1-6, Issue 10, Ukrainian SSR, Book 2, Part 5, Cloudiness, atmospheric phenomena, Gidrometeoizdat, Leningrad, 1990.

[20] V.V. Meshkov, Fundamentals of Lighting Engineering, Manual for universities, Part I, 2nd ed., Energiya, Moscow, 1979.

[21] B.A. Volynsky, Spherical trigonometry, Nauka, Moscow, 1977. 